\title{
Improved renal ischemia tolerance in females influences kidney transplantation outcomes
}

\author{
David D. Aufhauser Jr., Zhonglin Wang,' Douglas R. Murken, ${ }^{1}$ Tricia R. Bhatti, ${ }^{2}$ Yanfeng Wang, ${ }^{1}$ Guanghui Ge, ${ }^{1}$ \\ Robert R. Redfield III, ${ }^{3}$ Peter L. Abt, ${ }^{1,4}$ Liqing Wang, ${ }^{2}$ Nikolaos Svoronos, ${ }^{5}$ Arwin Thomasson, ${ }^{1}$ Peter P. Reese, ${ }^{6}$ \\ Wayne W. Hancock, ${ }^{2,7}$ and Matthew H. Levine ${ }^{1,4}$ \\ 'Department of Surgery, University of Pennsylvania, Philadelphia, Pennsylvania, USA. ²Department of Pathology and Laboratory Medicine, Children's Hospital of Philadelphia, Philadelphia, Pennsylvania, \\ USA. ${ }^{3}$ Department of Surgery, University of Wisconsin, Madison, Wisconsin, USA. " Department of Surgery, Children's Hospital of Philadelphia, Philadelphia, Pennsylvania, USA. ${ }^{5}$ The Wistar Institute, \\ Philadelphia, Pennsylvania, USA. ${ }^{6}$ Renal-Electrolyte and Hypertension Division and Department of Pathology and Laboratory Medicine, University of Pennsylvania, Philadelphia, Pennsylvania, USA.
}

\begin{abstract}
Experimentally, females show an improved ability to recover from ischemia-reperfusion injury (IRI) compared with males; however, this sex-dependent response is less established in humans. Here, we developed a series of murine renal ischemia and transplant models to investigate sex-specific effects on recovery after IRI. We found that IRI tolerance is profoundly increased in female mice compared with that observed in male mice and discovered an intermediate phenotype after neutering of either sex. Transplantation of adult kidneys from either sex into a recipient of the opposite sex followed by ischemia at a remote time resulted in ischemia recovery that reflected the sex of the recipient, not the donor, revealing that the host sex determines recovery. Likewise, renal IRI was exacerbated in female estrogen receptor $\alpha$-KO mice, while female mice receiving supplemental estrogen before ischemia were protected. We examined data from the United Network for Organ Sharing (UNOS) to determine whether there is an association between sex and delayed graft function (DGF) in patients who received deceased donor renal transplants. A multivariable logistic regression analysis determined that there was a greater association with DGF in male recipients than in female recipients. Together, our results demonstrate that sex affects renal IRI tolerance in mice and humans and indicate that estrogen administration has potential as a therapeutic intervention to clinically improve ischemia tolerance.
\end{abstract}

\section{Introduction}

Donor and recipient sex in human renal transplantation has been associated with differential short- and long-term allograft survival. Sex-based differences are ascribed to donor-recipient size mismatching and exposure to $\mathrm{H}-\mathrm{Y}$ minor histocompatibility (1-4). However, a focus on size- and immune-mediated effects has ignored the association between sex and ischemia-reperfusion injury (IRI).

The clinical manifestation of IRI in renal transplantation is delayed graft function (DGF), which is typically defined as a requirement for dialysis within the first week after transplantation (5). Although dialysis enables the support of patients until DGF resolves, DGF is associated with an increase in several untoward events including medical costs, length of hospital stay, rates of readmission after transplantation, rejection, and graft failure $(5,6)$. Therapies to mitigate IRI may lower DGF rates, thereby improving transplant outcomes and easing organ shortages by facilitating the use of organs with greater ischemic insult.

Sexual dimorphism with respect to tolerance of IRI has been noted in a number of laboratory animal models (7-11). Generally,

\section{Related Commentary: p. 1643}

Authorship note: D.D. Aufhauser Jr. and Z. Wang contributed equally to this work. Conflict of interest: The authors have declared that no conflict of interest exists. Submitted: September 16, 2015; Accepted: February 5, 2016.

Reference information: J Clin Invest. 2016;126(5):1968-1977. doi:10.1172/JCI84712. there is consensus that female sex conveys greater tolerance of, or protection from, IRI relative to the male sex. Investigations have focused on the effects of estrogen and testosterone on the intrinsic tissue tolerance of ischemia through differential expression of oxidative scavengers, HSPs, or other mediators (12-23). Moreover, a number of reports describe sex differences in the development of chronic injury patterns in animal transplant models $(15,24,25)$.

While sex disparities in IRI tolerance are accepted in animal systems, data pertaining to human renal transplantation are lacking. Likewise, significant attempts to use hormonal manipulation to achieve IRI tolerance have not been undertaken. To understand how such clinical interventions would be designed, we investigated several unresolved questions. First, does sex affect IRI tolerance via changes intrinsic to the kidney itself, via alterations in the host response to IRI, or both? Second, is protection from IRI in females due to beneficial effects of the hormonal environment, to impaired IRI tolerance in the male hormonal environment, or to a combination of these? Third, are these effects mutable with a change in hormonal background? And fourth, can we identify clear evidence of similar effects on IRI tolerance in clinical transplantation? These 4 points would assist in the identification of key information required to propose a translational investigation of IRI tolerance - namely, whether one would expect to see benefit with hormonal manipulation in human transplantation and whether one should propose to treat the donor, the donated organ, the recipient of the organ, or a combination of these. 


\section{Results}

Identification of differential tolerance of standardized renal IRI by sex. We have developed a standardized procedure for murine unilateral renal IRI with contralateral nephrectomy. Renal function was assessed daily for 4 days after IRI and was wholly attributable to the injured kidney. After a 28-day recovery period, mice were sacrificed and the degree of renal fibrosis quantitated using a computerized Sirius red scoring system (26). In this model, female C57BL/6 mice tolerated 28 minutes of warm IRI, with substantial but survivable injury that resulted in significant fibrosis $(n=10$, Figure 1A). However, male C57BL/6 mice subjected to the same 28-minute period of warm ischemia all developed renal failure and required sacrifice by 48 hours $(n=4)$. This led us to test what period of warm IRI in males would yield injury severity equivalent to that induced by 28 minutes of IRI in females. Warm IRI times of 18,20 , and 22 minutes in males led to nonsurvivable renal injury, whereas 15 minutes of warm renal IRI in male mice yielded injury equivalent to that induced by 28 minutes of warm IRI in female mice in terms of the blood urea nitrogen (BUN) rise and time to recovery ( $n=11$, Figure $1 \mathrm{~A})$ and fibrosis as quantified by Sirius red staining (mean \pm SEM of $10.6 \% \pm 5.1 \%$ in males after 15 minutes of IRI versus $8.1 \% \pm 3.9 \%$ in females after 28 minutes of IRI).

Effects of neutering on renal IRI tolerance. To further investigate sexual dimorphism, IRI tolerance was compared between neutered mice of each sex and hormonally intact controls. Mice were neutered at 3 weeks of age and, at 8 weeks of age, were subjected to renal IRI using the model outlined above. Neutered female mice had significantly impaired renal IRI tolerance with 28 minutes of warm renal IRI, as determined by the delayed recovery of renal function compared with that in normal female control mice ( $n=5$ in each group, $P<0.01$, Figure 1B). Fibrosis at 28 days was not significantly different (mean \pm SD of $8.6 \% \pm 2.8 \%$ in neutered females versus. $8.4 \% \pm 1.4 \%$ in control females). Because early renal functional differences exceeded those seen on fibrosis assessment, a second set of neutered and control mice were subjected to warm renal IRI and sacrificed 7 days after injury for assessment by quantitative PCR (qPCR) of the gene expression differences in the renal injury markers kidney injury markers 1 (KIM-1) and neutrophil gelatinase-associated lipocalin (NGAL). qPCR showed a significant increase in these renal injury markers in neutered females compared with that detected in the intact controls $(n=3$ per group, $P<0.001$, Supplemental Figure 1; supplemental material available online with this article; doi:10.1172/JCI84712DS1). Male neutered mice were subjected to 28 minutes of IRI and compared with neutered female and hormonally intact female controls. Neutered male mice $(n=5)$ survived 28 minutes of warm renal IRI, with renal impairment and functional recovery similar to what was observed in neutered female mice, but with more substantial renal injury than that observed in hormonally intact female mice $(P<0.05$, Figure $1 B)$. Their survival of 28 minutes of warm ischemia demonstrates a significant improvement in renal IRI tolerance relative to that of normal males. Twenty-eight days after injury, the kidneys of neutered male mice had fibrosis similar to that seen hormonally intact and neutered female mice when 28 minutes of warm renal IRI was used (mean \pm SEM of $10.2 \% \pm 1.4 \%$ in neutered males). These data indicate that the hormonal environment conveys differential tolerance of renal IRI, with a female hormonal environment providing significant protection and a male hormonal environment being associated with worse injury.

Transplantation and subsequent warm renal IRI. To examine the mutability of these effects, kidneys were directly transplanted between $\mathrm{C} 57 \mathrm{BL} / 6$ mice with the standardized minimal cold ischemia time (30 minutes), using female donors to female recipients $(\mathrm{F} \rightarrow \mathrm{F})$, male donors to female recipients $(\mathrm{M} \rightarrow \mathrm{F})$, and female donors to male recipients $(\mathrm{F} \rightarrow \mathrm{M})$, and then native nephrectomies were performed 5 days after transplantation. We allowed 4 weeks for functional recovery of the transplanted kidneys and, upon testing, confirmed normal renal function (normal BUN) in all mice. Mice were then subjected to 25 minutes of warm IRI of the transplanted kidney. The $\mathrm{F} \rightarrow \mathrm{F}$ and $\mathrm{M} \rightarrow \mathrm{F}$ cohorts showed universal survival ( 5 of 5 and 5 of 5 , respectively) and similar impairment of renal function during the first 96 hours of IRI (Figure 1C). The $\mathrm{F} \rightarrow \mathrm{M}$ group had significantly decreased survival rates (2 of $5, P=0.03$ ) and significantly higher BUN curves in the survivors compared with survival rates for the other groups $(P<0.0001)$. Transplanted kidneys were collected 28 days after IRI to assess fibrosis. Given the high mortality rate observed in the $\mathrm{F} \rightarrow \mathrm{M}$ group, transplantations were performed in a second group of $\mathrm{F} \rightarrow \mathrm{M}$ mice that underwent 25 minutes of warm IRI of the transplanted kidney, without native nephrectomy, to allow long-term survival and collection of the transplanted kidney for histological examination. The $\mathrm{F} \rightarrow \mathrm{F}$ and $\mathrm{M} \rightarrow \mathrm{F}$ cohorts demonstrated similar fibrosis at 28 days (mean \pm SEM of $8.6 \% \pm 0.9 \%$ in $\mathrm{F} \rightarrow \mathrm{F}$ versus $6.9 \% \pm 1.4 \%$ in $\mathrm{M} \rightarrow \mathrm{F}, P=0.29$, Figure 1, D and $\mathrm{E}$ ). The $\mathrm{F} \rightarrow \mathrm{M}$ transplant group had significantly more fibrosis than did the other 2 transplant groups (mean \pm SD of $18.3 \% \pm 1.8 \%, P<0.001$, Figure 1 , D and E). Tolerance of renal IRI was associated with the hormonal environment and was not renal intrinsic.

Role of estrogen receptors. To assess the role of the estrogen receptor in determining the renal IRI phenotype, we compared renal injury following 28 minutes of warm IRI in WT and estrogen receptor $\alpha-\mathrm{KO}(\mathrm{ER} \alpha-\mathrm{KO})$ female mice. $\mathrm{ER} \alpha-\mathrm{KO}$ mice showed significantly increased vulnerability to renal IRI compared with that seen in controls, as evidence by increased post-injury BUN levels ( $n=5$ in each group, $P<0.01$, Figure $2 \mathrm{~A}$ ). The standardized injury led to universal death of $\mathrm{ER} \alpha-\mathrm{KO}$ mice by postoperative day 4 , whereas all control mice survived out to day $28(P<0.01)$. These results demonstrate that the estrogen receptor is required for improved female tolerance of renal IRI.

Effects of supplemental estrogen therapy. We next investigated the potentially translatable benefits of estrogen manipulation in renal IRI. Female mice received 2 doses of estrogen (17 $\beta$-estradiol, $1 \mathrm{mg} / \mathrm{kg}$ i.p.) or vehicle at 16 hours and 1 hour before undergoing 28 minutes of warm renal IRI. Estrogen-treated females had significant improvement in post-injury renal function compared with that observed in controls $(n=5$ in each group, $P<0.01$, Figure $2 \mathrm{~B}$ ), as well as decreased fibrosis at 28 days (mean \pm SEM of $5.6 \% \pm 0.3 \%$ in treated animals versus $8.2 \% 1.7 \%$ in controls, $P=0.01$, Figure 2, C and D). A small pilot group of hormonally intact male mice treated with the same dose of estrogen $(1 \mathrm{mg} /$ kg i.p.) showed no benefit. A larger cohort of hormonally intact males treated with a higher dose of estrogen $(10 \mathrm{mg} / \mathrm{kg}, n=5)$ and subjected to 15 minutes of warm renal IRI showed renal injury 
A
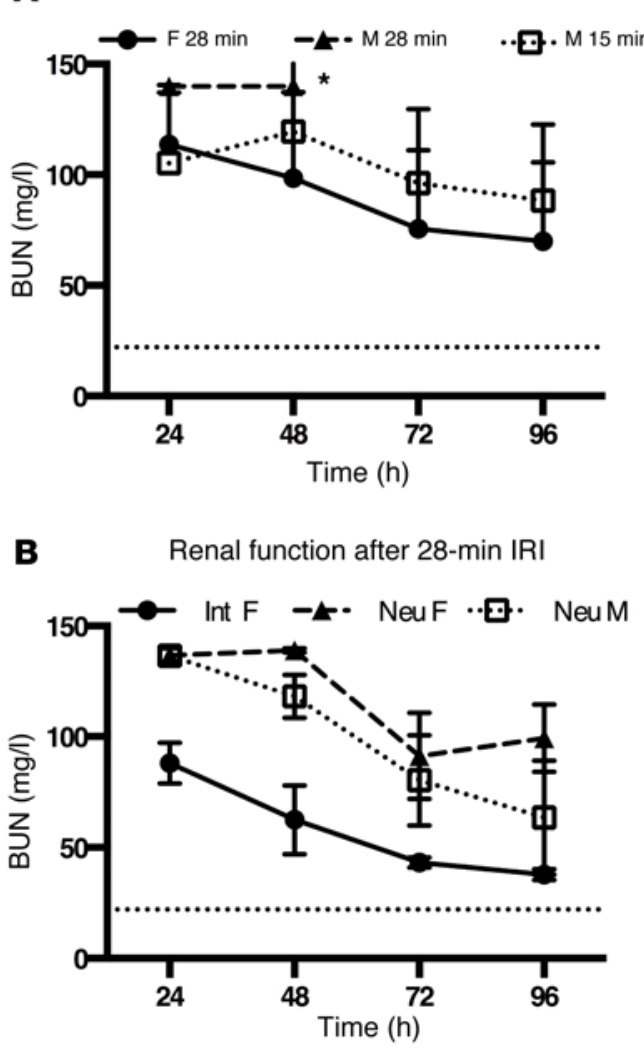

D
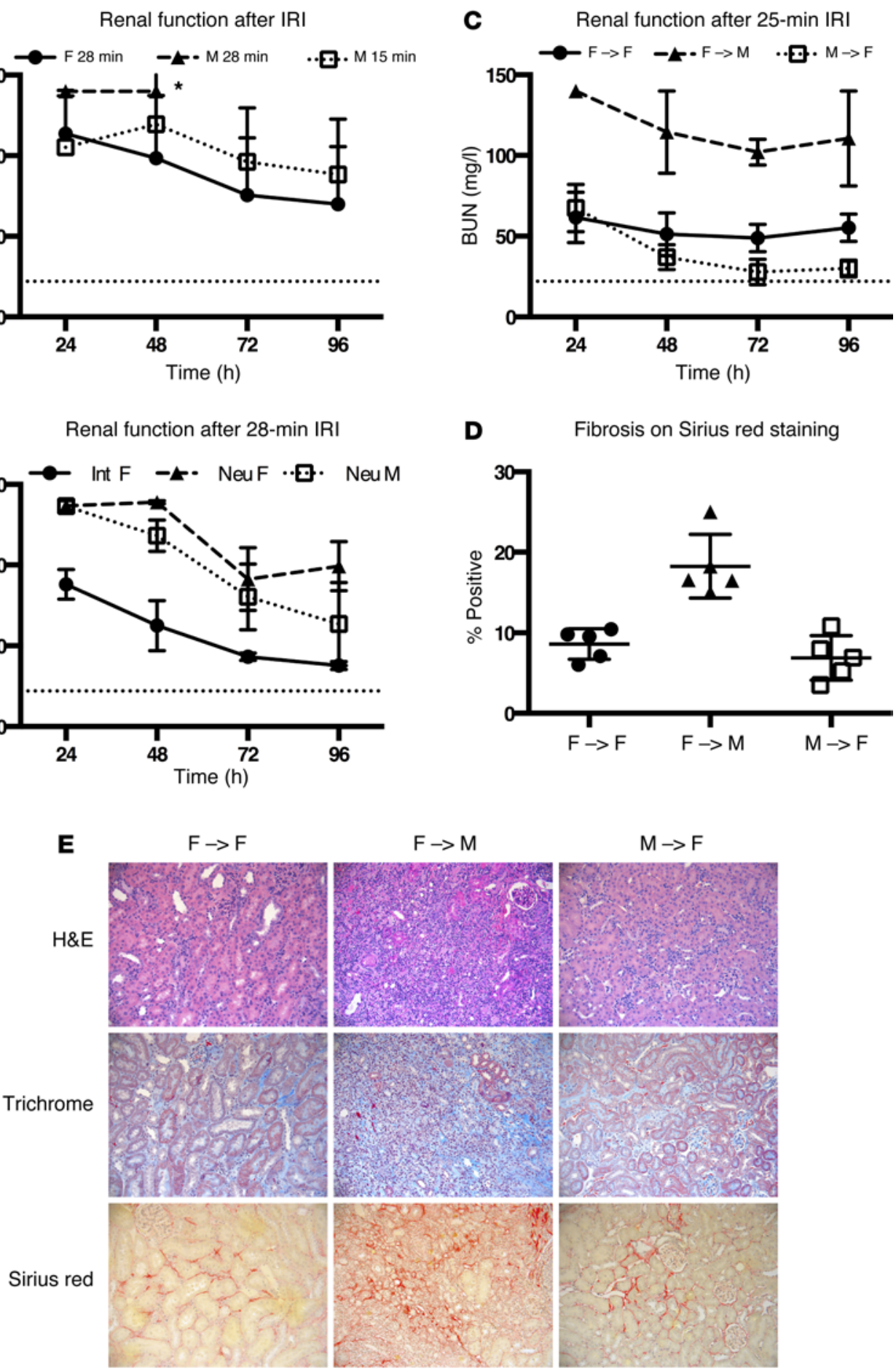

Figure 1. Female hormonal environment protects against renal IRI. (A) BUN measurements after 28 minutes of renal IRI in female mice (F $28 \mathrm{~min} ; n=10$ ) showed renal injury equivalent to that in male mice following 15 minutes of renal IRI (M 15 min; $n=11 ; P=$ 0.36 , by 2 -way ANOVA); 28 -minute IRI was unsurvivable in male mice (M $28 \mathrm{~min}^{*} n=4$ ); dashed line shows the baseline BUN level. (B) Neutered female mice (Neu F; $n=$ 5) had impaired renal function after warm IRI compared with that seen in intact females (Int F; $n=5 ; P<$ 0.01 , by 2-way ANOVA). Neutered male mice (Neu M; $n=5$ ) tolerated IRI with impairment similar to that in neutered females, but worse function than in intact females $(P<$ 0.01 , by 2 -way ANOVA). (C) Female kidneys transplanted into female recipients $(\mathrm{F} \rightarrow \mathrm{F} ; n=5)$ and male kidneys transplanted into female recipients (M $\rightarrow F ; n=5$ ) had similar impairment of renal function when subjected to 25 minutes of warm IRI. Female kidneys transplanted into male recipients $(F \rightarrow M ; n=$ 5) had decreased survival (2 of 5), with significantly higher BUN levels compared with those of either of the other 2 groups $(P<0.0001$, by 2 -way ANOVA). (D) The $\mathrm{F} \rightarrow \mathrm{M}$ transplanted kidneys had significantly more fibrosis on Sirius red staining at 28 days than did the transplanted kidneys in either of the other 2 groups $(P<0.001$, by 1 -way ANOVA). (E) H\&E, trichrome, and Sirius red staining (original magnification, $\times 20$ ) showed increased fibrosis in the $\mathrm{F} \rightarrow \mathrm{M}$ cohort compared with that observed in the $\mathrm{F} \rightarrow \mathrm{F}$ and $\mathrm{M} \rightarrow \mathrm{F}$ cohorts. Error bars indicate the mean \pm SEM. similar in extent to that seen in vehicle-treated controls $(n=5$, Figure 2E). However, neutered male mice treated with the same dose of estrogen $(10 \mathrm{mg} / \mathrm{kg}, n=5)$ demonstrated a trend toward protection from renal injury after 28 minutes of warm renal IRI compared with that observed in neutered male, vehicle-treated controls $(P=0.08, n=5$, Figure $2 \mathrm{~F})$.

To assess whether a similar benefit could be achieved with treatment after ischemic injury, hormonally intact female mice were treated with 2 doses of vehicle or higher-dose estrogen (10 mg/kg i.p.) at laparotomy closure and 24 hours after reper- fusion. Estrogen-treated mice showed a trend toward improved renal function, but it was not significant $(n=5$ per group, $P=0.14$, Figure $2 G$ ). No difference in fibrosis was observed by Sirius red staining (mean \pm SEM of $4.1 \% \pm 0.5 \%$ in controls versus $4.2 \% \pm$ $0.6 \%$ in treated mice).

Cold ischemia transplantation model to identify intrinsic and extrinsic hormonal effects. We next studied whether these effects reflected an intrinsic tolerance of the kidney to ischemia, whether they were due to alterations in the host's response to ischemia, or both. This distinction cannot be made in a single animal model of 


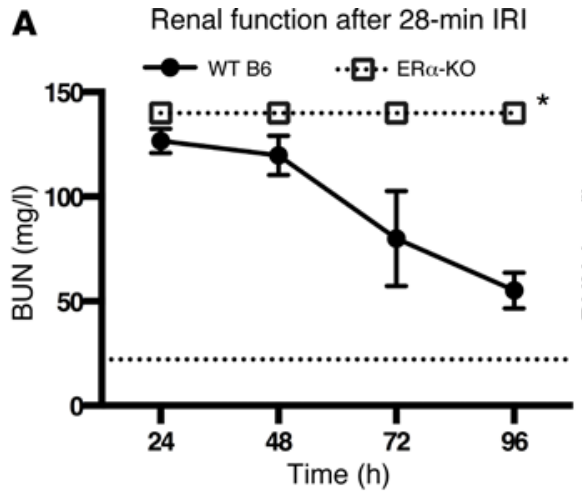

B Renal function after 28-min IRI

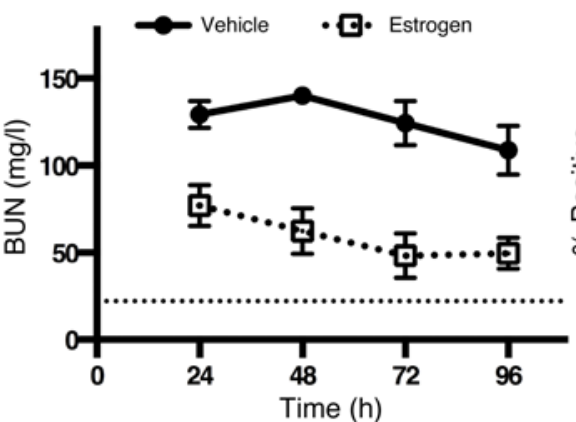

$\mathbf{F}$
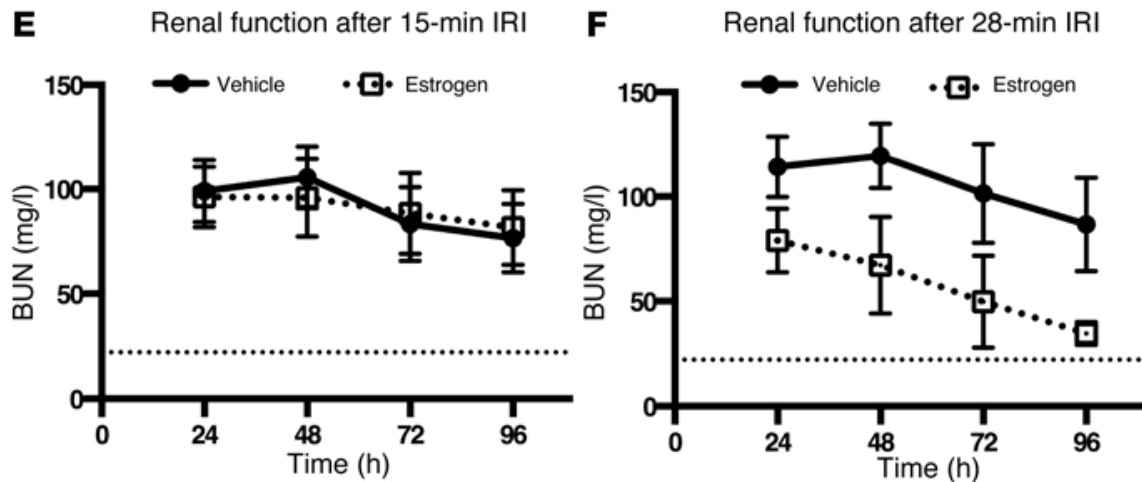

C

Fibrosis on Sirius red staining
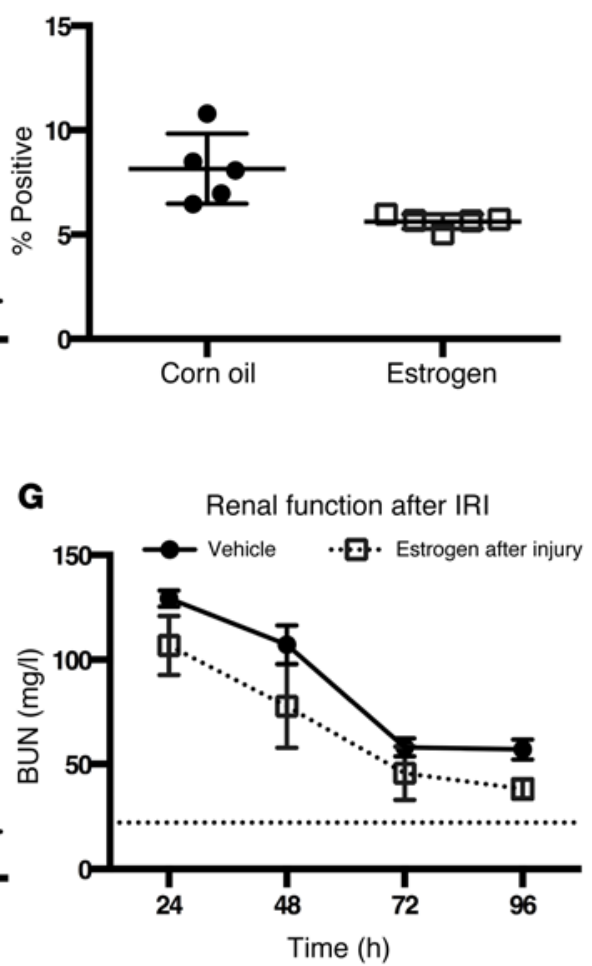

Figure 2. Role of estrogen in murine renal IRI. (A) ER $\alpha-K O$ mice $(n=5)$ had elevated BUN levels after warm IRI compared with levels in WT B6 controls ( $n=5 ; P<0.01$, by 2 -way ANOVA). This injury was universally lethal in $\mathrm{ER} \alpha-\mathrm{KO}$ mice by 96 hours after injury, as denoted by the asterisk. (B) Female mice treated with supplemental estrogen $(1 \mathrm{mg} / \mathrm{kg}$ at 16 hours and 1 hour before IRI; $n=5$ ) and subjected to warm IRI had lower post-injury BUN levels compared with levels in vehicle-treated controls $(n=5 ; P<0.01$, by 2-way ANOVA). (C) Sirius red fibrosis scoring at 28 days was significantly decreased in the estrogen-treated arm ( $P=0.01$, by 2-tailed Student's $t$ test). (D) H\&E, trichrome, and Sirius red staining (original magnification, $\times 20$ ) showed decreased fibrosis at 28 days in the estrogen-treated cohort. (E) Hormonally intact male mice treated with high-dose supplemental estrogen $(10 \mathrm{mg} / \mathrm{kg}$ at 16 hours and 1 hour before IRI; $n=5$ ) and subjected to warm IRI had equivalent post-injury BUN levels compared with those of vehicle-treated controls ( $n=5 ; P=0.98$, by 2-way ANOVA). (F) Neutered male mice treated with high-dose supplemental estrogen ( $10 \mathrm{mg} / \mathrm{kg}$ at 16 hours and 1 hour before IRI; $n=5$ ) and subjected to warm IRI had lower post-injury BUN levels compared with those of vehicle-treated controls ( $n=4 ; P=0.08$, by 2-way ANOVA). (C) Post-injury administration of estrogen to intact females $(10 \mathrm{mg} / \mathrm{kg}$ at surgical closing and at 24 hours) resulted in a nonsignificant trend toward improved post-injury BUN levels compared with those of vehicle-treated controls ( $n=5$ per group; $P=0.14$, by 2-way ANOVA). Error bars indicate the mean $\pm \mathrm{SEM}$. warm IRI, so we developed a murine renal transplantation model with a standardized cold ischemic time. This procedure led to early organ dysfunction, so, to allow for renal recovery, the native kidneys were left in situ for 5 days and then removed. Renal function was measured 2 and 9 days after native nephrectomy to reflect the performance of the transplanted kidney, and transplanted kidney tissue was assessed for fibrosis 28 days after transplantation.

In this model, syngeneic transplantation can be done with a tolerance of 8 hours of cold storage of the kidney between procurement and transplantation. This degree of cold ischemia led to significant but recoverable injury as assessed by BUN levels after native nephrectomy and subsequent fibrosis formation (Figure 3, A and B). Longer periods of cold IRI led to a less-consistent survival that was most evident in groups other than the $\mathrm{F} \rightarrow \mathrm{F}$ group, so the 8 hours of cold ischemic injury was used. To assess whether donor or recipient sex was determinative of renal cold IRI tolerance, we performed the transplantation experiments using $\mathrm{F} \rightarrow \mathrm{F}$ mice $(n=5), \mathrm{M} \rightarrow \mathrm{F}$ mice $(n=5), \mathrm{F} \rightarrow \mathrm{M}$ mice $(n=6)$, and $\mathrm{M} \rightarrow \mathrm{M}$ mice $(n=6)$. The $\mathrm{F} \rightarrow \mathrm{F}$ cohort showed superior function of the transplanted kidney, followed by the $\mathrm{F} \rightarrow \mathrm{M}$ cohort, the $\mathrm{M} \rightarrow \mathrm{M}$ cohort, and the $\mathrm{M} \rightarrow \mathrm{F}$ cohort (Figure $3 \mathrm{~A})$. The difference was significant between the $\mathrm{F} \rightarrow \mathrm{F}$ and $\mathrm{M} \rightarrow \mathrm{F}$ cohorts $(P<0.05)$. Renal fibrosis was 


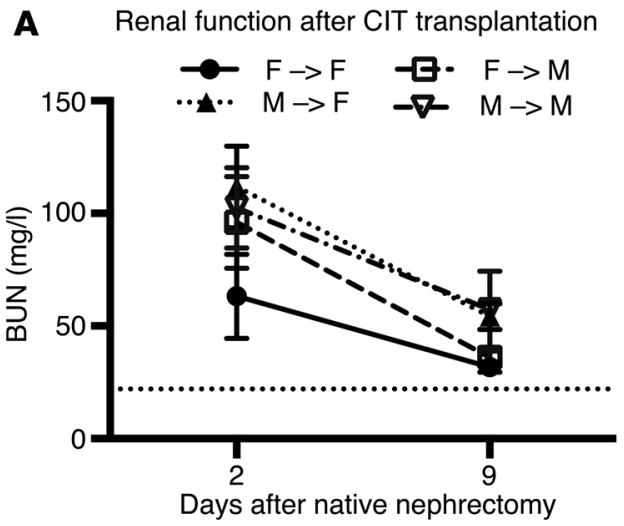

Figure 3. Sex environment affects a transplant model of cold renal IRI. (A) Murine kidneys transplanted with an 8-hour period of cold ischemia from female donors into female recipients $(F \rightarrow F ; n=5)$ had superior renal function after native nephrectomy compared with that seen in male donor kidneys transplanted into female recipients ( $M \rightarrow F ; n=5 ; P<0.05$, by 2-way ANOVA) and had a trend toward improved function compared with that of female donor kidneys transplanted into male recipients $(F \rightarrow M ; n=6)$ and male donor kidneys transplanted into male recipients $(M \rightarrow M ; n=6)$. CIT, cold ischemia time. (B) Renal fibrosis on Sirius red staining showed a trend toward increased fibrosis in the $\mathrm{M} \rightarrow \mathrm{F}$ cohort that was significant compared with the fibrosis observed in all other cohorts $(P<0.001$, by 1 -way ANOVA). Error bars indicate the mean \pm SEM. increased in the $\mathrm{M} \rightarrow \mathrm{F}$ cohort compared with that seen in all other cohorts $(P<0.001$, Figure $3 \mathrm{~B})$. These results indicate that female donors confer a protective IRI phenotype in mice.

Influence of sex on renal IRI in human kidney transplant recipients. DGF is the functional result of IRI in clinical renal transplantation and is defined as a requirement for dialysis within 7 days of transplantation (5). Given the sexual dimorphism of renal IRI tolerance observed in mice, we postulated that female sex would be protective against DGF and that donor-recipient size discrepancy would not account for these differences, as has been previously considered (1). To examine this hypothesis, we analyzed deceased donor renal transplants from United Network for Organ Sharing (UNOS) national registry data from 2009 to 2014, with DGF as the outcome of interest using uni- and multivariable Cox regression analysis. Since DGF is challenging to identify in patients who are not on renal replacement therapy because of residual renal function in native kidneys, we included in our analysis only adult recipients who were on dialysis prior to transplantation. Additionally, we excluded transplants from donors weighing less than $40 \mathrm{~kg}$ or under 10 years of age, since such donors were unlikely to have entered puberty, and the donor kidneys were unlikely to have the characteristic hormone profiles of either sex.

Our analysis included 46,691 renal transplants. The overall characteristics of the transplants with and without DGF are shown in Supplemental Table 1. On univariate analysis, male recipients had a significantly higher DGF rate of $29.2 \%$ compared with $23.2 \%$ for female recipients $(P<0.001)$. Grafts from male donors also had a higher DGF rate of $27.7 \%$ versus $25.7 \%$ for grafts from female donors $(P=0.012)$. On multivariable analysis, male donor sex remained modestly associated with increased DGF (odds ratio [OR], 1.10; 95\% CI, 1.05-1.16), but male recipient sex was strongly associated with DGF (OR, 1.39; 95\% CI, 1.33-1.46) (Table 1). The magnitude of the interaction between recipient sex and DGF was not as large as the extended criteria donor (ECD) status (OR, 1.45; adjusted for size mismatch in multivariable regression for outcome of DGF. Male recipient sex remained signicantly associated with DGF (OR, 1.38; $95 \%$ CI, 1.31-1.46), and the adjusted OR associated with recipient sex was similar in magnitude to the unadjusted OR. Next, we examined whether size mismatch modified the effect of recipient sex on DGF. We found that the interaction terms between recipient sex and size mismatch were nonsignificant. We fit 10 additional multivariable models stratified by size-mismatch deciles. Both extremes of size mismatch were associated with increased DGF risk (Figure 4). In all deciles, male recipient sex remained significantly associated with DGF $(P<0.01$ in all deciles $)$. These data indicate that a donor-recipient size mismatch does not modify the effect of recipient sex on DGF rates or account for the increased DGF risk in male recipients.

Influence of sex after menopause. To estimate the relative influence of recipient estrogen exposure on human sex effects, we performed a second multivariate analysis of recipients over the age of 65 years, as this threshold assures postmenopausal female recipients; 7,788 recipients were in this subgroup (Table 3). The OR for male recipients in this cohort was 1.26 (95\% CI, 1.12-1.41), a reduction from 1.39 in the larger group that suggests a possible partial loss of female protection from IRI within this subgroup, although the effects of increased donor age or donor risk index may also play a role.

Paired recipients of different sexes. To confirm our findings of a significant recipient sex effect, we limited our analysis to those who donated both kidneys, with 1 allocated to a recipient of each sex. This assured that all donor attributes were identical between recipient pairs, except for the cold ischemia time (which was included in our multivariable analysis), and included 16,954 recipients (Table 3 ). Within this subgroup of donors, male recipient sex was again associated with higher rates of DGF (OR, 1.43; 95\% CI, 1.34-1.53). The magnitude of this effect was once again larger than the effect of size mismatch 
Table 1. Multivariate analysis of variables associated with DGF in 46,691 deceased donor kidney transplants between 1997-2011

\begin{tabular}{|c|c|c|c|}
\hline Characteristic & $\mathrm{OR}$ & SEM & $95 \% \mathrm{Cl}$ \\
\hline \multicolumn{4}{|c|}{ Sex } \\
\hline Male donor & 1.10 & 0.03 & $1.05-1.16$ \\
\hline Male recipient & 1.39 & 0.03 & $1.33-1.46$ \\
\hline \multicolumn{4}{|c|}{ Recipient age (yr) } \\
\hline $18-40$ & REF & & \\
\hline $41-55$ & 1.18 & 0.04 & $1.11-1.26$ \\
\hline $56-69$ & 1.25 & 0.04 & $1.18-1.34$ \\
\hline $70+$ & 1.33 & 0.07 & $1.20-1.47$ \\
\hline \multicolumn{4}{|c|}{ Recipient race } \\
\hline White & REF & & \\
\hline Black & 1.60 & 0.04 & $1.52-1.68$ \\
\hline Hispanic & 1.34 & 0.04 & $1.26-1.43$ \\
\hline Asian/other & 1.22 & 0.06 & $1.11-1.34$ \\
\hline Other & 1.35 & 0.10 & $1.16-1.57$ \\
\hline Black donor & 0.97 & 0.03 & $0.91-1.04$ \\
\hline ECD & 1.45 & 0.05 & $1.36-1.54$ \\
\hline $\mathrm{DCD}$ & 2.45 & 0.08 & $2.30-2.60$ \\
\hline Recipient DM & 1.07 & 0.03 & $1.02-1.13$ \\
\hline Right kidney & 1.11 & 0.02 & $1.07-1.16$ \\
\hline \multicolumn{4}{|c|}{ Cause of ESRD } \\
\hline Other & REF & & \\
\hline GN & 0.91 & 0.03 & $0.85-0.97$ \\
\hline HTN & 0.94 & 0.03 & $0.89-1.00$ \\
\hline \multicolumn{4}{|c|}{ PRA } \\
\hline 0 & REF & & \\
\hline 1-19 & 1.02 & 0.04 & $0.94-1.11$ \\
\hline 20-79 & 0.92 & 0.03 & $0.86-0.98$ \\
\hline $80+$ & 1.06 & 0.04 & $0.99-1.13$ \\
\hline \multicolumn{4}{|c|}{ CIT } \\
\hline$<8 \mathrm{~h}$ & REF & & \\
\hline $8-16 h$ & 1.13 & 0.04 & $1.06-1.20$ \\
\hline $17-24 \mathrm{~h}$ & 1.49 & 0.05 & $1.39-1.60$ \\
\hline $25-48 \mathrm{~h}$ & 1.88 & 0.07 & $1.74-2.03$ \\
\hline $49+$ & 0.78 & 0.13 & $0.57-1.07$ \\
\hline Missing & 1.10 & 0.09 & $0.94-1.29$ \\
\hline
\end{tabular}

DM, diabetes mellitus; ESRD, end-stage renal disease; GN, glomerulonephritis; HTN, hypertension; REF, reference group (OR, 1.00 for the reference group).

in any of the 10 size-match deciles (maximum OR, 1.18 in decile 10; 95\% CI, 1.06-1.32), of recipient diabetes status (OR, 1.09; 95\% CI, 1.01-1.18), or of PRA levels above 80\% (OR, 1.15; 95\% CI, 1.05-1.27). The magnitude of effect was similar to that of ECD status (OR, 1.51; 95\% CI, 1.36-1.67), but smaller than that of DCD status (OR, 2.40; 95\% CI, 2.18-2.64).

Outcomes after DGF. We assessed whether recipient sex is associated with graft survival after DGF. Both male and female renal graft recipients with DGF exhibited decreased death-censored graft survival compared with that observed in recipients of the same sex without DGF $(P<0.01$, Figure 5$)$. Male and female renal transplant recipients who did not develop DGF had equivalent graft survival rates $(P=0.20)$. Female renal transplant recipients with DGF had decreased graft survival compared with that of male recipients with DGF $(P=0.002)$, although the overall magnitude of this difference was small.

\section{Discussion}

We undertook a systematic examination of sexual dimorphism in renal IRI outcomes, with the aim of assessing possible opportunities for intervention to reduce injury in renal transplantation or in other settings of ischemia. The magnitude of IRI protection observed in C57BL/6 female mice was large, with females tolerating nearly twice the period of ischemia as males to attain an equivalent injury, consistent with the differential renal IRI tolerance described in other murine backgrounds (14). Since small differences in timing or temperature of renal IRI convey substantial differences in injury in the mouse model, a near-doubling of the duration of renal IRI tolerance is remarkable.

Sex differences in renal ischemia in mice have been reported previously, but our work expands substantially on these findings. Of particular relevance are the studies of Park et al. and Muller et al. that attributed much of the observed sex difference in IRI tolerance to the detrimental effects of androgens (15-18). These authors reported superior renal IRI tolerance in orchiectomized male rodents compared with that in hormonally intact controls, which our experiments confirm. Although administration of dihydrotestosterone (DHT) or testosterone over 14 days was reported to reverse the protected phenotype of orchiectomized males and increase renal injury in females, experiments with shorter-term androgen administration to females by Park et al. (30 minutes) and Muller et al. (7 days) did not lead to increased IRI vulnerability $(15,17)$. The increased susceptibility induced by androgens appears to require long-term exposure, making blockade of this axis less promising for clinical translation.

Our effort builds on this earlier work by focusing on the elements of sex effects that are relevant and modifiable in a time frame compatible with clinical transplantation and by demonstrating that the murine findings of sex differences extend to humans. To this end, we wish to emphasize several key findings of our work. First, we performed a series of unique transplantation experiments to establish that greater female tolerance of renal IRI is extrinsic to the kidney. Transplantation of adult kidneys across sexes, with subsequent IRI, showed that renal IRI tolerance is mutable to that of the new environment and therefore potentially modifiable in transplantation. Second, we demonstrated that female protection extends to cold ischemia as well as warm ischemia. This finding has particular relevance in transplantation and, to our knowledge, has not previously been reported. Third, we showed, using several approaches, that sex impacts human DGF, that this effect is large, that this effect is smaller in postmenopausal-aged recipients, and, importantly, that this difference is not explained by a mismatch in donor and recipient sizes or by nephron dosing. While previous reports have examined sex effects on long-term human renal transplant outcomes, none has previously examined short-term IRI-related outcomes such as DGF. DGF was associated with extremes in size mismatches, not just in large recipients of kidneys from small donors, likely attributable to nephron dosing, but also in small (nonpediatric) recipients of large donor kidneys. The cause of this is not defined 
Table 2. Association of DGF with size mismatch by decile

\begin{tabular}{|lcccc|}
\hline & BW ratio & OR & SEM & 95\% Cl \\
\hline $\begin{array}{l}\text { Donor larger; } \\
\text { recipient smaller }\end{array}$ & Decile 1 & 1.17 & 0.06 & $1.07-1.29$ \\
& Decile 2 & 1.10 & 0.05 & $1.01-1.21$ \\
\hline & Decile 3 & 0.98 & 0.05 & $1.01-1.21$ \\
\hline & Decile 4 & 1.03 & 0.05 & $0.93-1.12$ \\
\hline & Decile 5 & REF & & \\
\hline & Decile 6 & 1.02 & 0.05 & $0.93-1.12$ \\
\hline & Decile 7 & 1 & 0.05 & $0.91-1.10$ \\
\hline Decile 8 & 1 & 0.05 & $0.91-1.10$ \\
\hline $\begin{array}{l}\text { Donor smaller; } \\
\text { recipient larger }\end{array}$ & Decile 9 & 1.11 & 0.05 & $1.01-1.21$ \\
\hline
\end{tabular}

46,691 deceased donor kidney transplant recipients were stratified into deciles by donor/recipient BW ratio to identify size discrepancy effects on DGF occurrence. Both extremes of size mismatch were associated with increased risk of DGF.

by this analysis, but we can hypothesize that initial hypoperfusion of a large kidney with a smaller circulating volume, or constraint of the kidney in a smaller retroperitoneal space, could be contributing factors. Fourth, we demonstrated that both donor and recipient hormonal milieus contribute to renal IRI tolerance; recipient effects are dominant in human transplant outcomes, while donor effects appear somewhat stronger in mice. This distinction between donor and recipient effects is critical in determining whether potential human hormonal intervention would need to be undertaken in the donor or in the recipient in transplant scenarios. Fifth, we showed that supplemental $17 \beta$-estradiol administered over a short time course prior to IRI is protective in female mice and in neutered, but not hormonally intact, males. These data provide a rationale for studies in larger animals and, potentially, for human interventional trials of estrogen administration in female patients in settings of predictable exposure to renal ischemia.

Therapeutic intervention driven by the increased IRI tolerance of females depends on establishing that sex effects are mutable with alterations to a kidney's hormonal milieu. Our intersex renal transplants established that kidneys of either sex assume the IRI phenotype of the recipient when exposed to a new hormonal environment for at least 2 weeks. As donors in this experiment were fully developed adult mice, the differential tolerance of IRI is not an intrinsic kidney property.

Multiple authors have described protective actions of estrogens in IRI in both male and female rodents, although in most reports the magnitude of this effect was smaller than that seen with the removal of testosterone $(15,27,28)$. Moreover, renoprotection by estrogen is described in other forms of renal injury, including rat models of chronic allograft nephropathy (CAN) $(15,24,25)$, age-related glomerular damage (29), and hypertensive nephrosclerosis (30). The role of estrogen receptors in this process remains a subject of debate. Previous reports concluded that the benefit of estrogen in ischemia was independent of estrogen receptors, but these experiments were conducted in cardiac arrest models with multiple variables at play rather than in a highly controlled renal ischemia model $(27,28)$. The universal lethality observed after 28 minutes of warm ischemic injury in $\mathrm{ER} \alpha-\mathrm{KO}$ mice in our series reflects a more extreme phenotype than that achieved with surgical neutering and suggests that this receptor plays an important mechanistic role.

The mutability of the IRI phenotype is reinforced by the decreased renal injury seen in female mice with short-term estrogen supplementation beginning 16 hours before injury. This time course can feasibly be translated to transplantation, whereby the majority of organs are recovered on short notice from unanticipated donors. The ability to administer $\beta$-estradiol or any medication to reduce renal IRI in a transplantation setting depends on the persistence of its beneficial effects or, at a minimum, the lack of detrimental effects in the procurement of other organs. Sex differences in IRI tolerance are well described in cardiac tissue $(7,31,32)$ and the nervous system $(8,33)$, and short-term administration of $17 \beta$-estradiol directly improved IRI tolerance in rat livers (9) and myocardium (34) when given 1 and 3 hours before injury, respectively, making nonrenal off-target effects of lesser concern.

Our analysis of the UNOS data underscores the idea that sexual dimorphism in renal transplant-associated IRI is a significant, previously undescribed event in humans, too. Female sex afforded protection from IRI, with both female donor and female recipient sex associated with lower rates of DGF. In recipients, the magnitude of the effect was large, with a stronger association found between male sex and DGF (OR, 1.39) than between DGF and

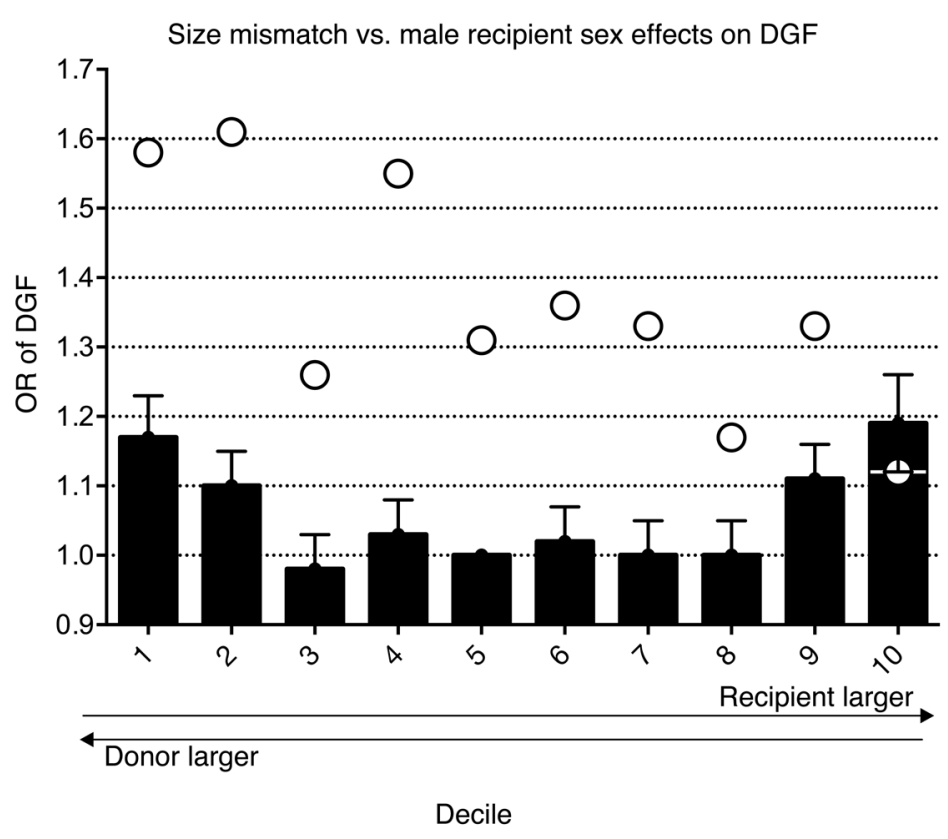

Figure 4. Size discrepancy in human renal transplantation. Deceased donor renal transplants ( $n=46,691)$ were stratified into deciles on the basis of donor/recipient BW ratio and analyzed for association with DCF. The extremes of size mismatch on both ends of the curve were associated with increased DGF risk (bars). The magnitude of these effects on DGF was smaller than recipient sex effects in every decile except the smallest donor/recipient ratio (circles). 
Table 3. OR for DGF in male recipients of kidney transplants in selected subgroups

$\begin{array}{lcccc}\text { Subgroup } & \boldsymbol{n} & \text { OR } & \text { SEM } & \text { 95\% Cl } \\ \begin{array}{l}\text { Age }>65 \mathrm{yr} \\ \text { (postmenopausal) }\end{array} & 7,788 & 1.26 & 0.07 & 1.12-1.41 \\ \begin{array}{l}\text { Donor with 1 male } \\ \text { and 1 female graft } \\ \text { recipient }\end{array} & 16,954 & 1.43 & 0.05 & 1.34-1.53 \\ & & & & \end{array}$

recipient diabetes or a PRA level over $80 \%$, but a weaker association between ECD or DCD donor status and DGF. The true magnitude of this association may be even larger; an OR of 1.43 was observed when the analysis was limited to the smaller but better-controlled subgroup of matched donor kidney pairs allocated to 1 male and 1 female recipient. The effect from donor sex was smaller (OR, 1.10), a notable difference from our findings in mice, in which the preischemic hormonal environment of the recipient appeared to have a stronger effect on IRI tolerance than did donor sex. The reduction in the association of male recipient sex with DGF (OR, 1.26) observed in the subgroup analysis of patients over 65 years of age, however, suggests that human sex effects might demonstrate similar mutability in the face of alterations in the hormonal environment, as we observed in mice, although the effect of aging or other donor-recipient matching factors in the elderly, independent of hormonal effect, must also be considered. In total, these studies encourage the use of estrogen administration to female recipients as a reasonable starting point for interventional approaches in larger animal models or pilot clinical trials.

No previous reports to our knowledge have focused on the link between sex and DGF in clinical renal transplantation, though there are studies focused on the role of sex in long-term outcomes. A large, retrospective study identified lower rates of graft failure at 1 and 10 years in female recipients, but higher rates of graft loss with female donors at identical time points (2). The worst-performing cohort involved grafts from male donors transplanted into female recipients, an effect the authors attributed to $\mathrm{H}-\mathrm{Y}$ incompatibility, although no direct evidence was provided (2). This observation was confirmed by data from the US Renal Data System (USRDS) showing increased rates of graft failure in recipients of male grafts at 1 but not 10 years (4). A single-center study agreed with this finding, but noted that the effect was concentrated in male donors of smaller BMI (1). As DGF rates were not specifically examined in these studies, it is unclear whether their findings have any significance with regard to IRI or how short-term manipulations of sex hormones might affect these outcomes.

In summary, sex differences in renal IRI tolerance in mice are both large and mutable with hormonal modulation. In human kidney transplantation, recipient sex significantly influences DGF rates, and this is not explained by BW mismatch or nephron-dosing effects. This work justifies further investigation into hormonal intervention as a renal protective strategy against IRI in larger animals and humans.

\section{Methods}

Animals. We used 8- to 12-week-old WT C57BL/6 adult mice (The Jackson Laboratory) or ER $\alpha-\mathrm{KO}$ mice on a C57BL/6 background (The Wistar Institute) and weighing 18-25 g.

Warm IRI model. Mice were anesthetized with prewarmed pentobarbital sodium $(65 \mathrm{mg} / \mathrm{kg} \mathrm{IP})$. Immediately after loss of a righting reflex, they were placed on a heated surgical pad $\left(37^{\circ} \mathrm{C}\right)$ in a temperature-controlled operative apparatus. Core body temperature was continuously measured throughout and maintained at $36.0^{\circ} \mathrm{C} \pm 0.5^{\circ} \mathrm{C}$. Using an operating microscope, an abdominal midline incision was made, and the left renal pedicle was exposed and clamped for $28 \mathrm{~min}$ utes with a microvascular clip (Roboz Surgical Instrument Co.). After the clamp was released, the right kidney was exposed and removed, and the abdomen was closed. Animals were s.c. injected with 100 $\mathrm{ml} / \mathrm{kg}$ warm saline after the operation to assist in the maintenance of hydration. Animals were kept in an incubator $\left(37^{\circ} \mathrm{C}\right)$ from the time of anesthetic administration until completely awake (26).

Neutering. Mice at 3 weeks of age underwent ovariectomy or castration under inhalation anesthesia (isoflurane).

Renal transplantation. Murine donor nephrectomy was performed under anesthesia with pentobarbital sodium (65 mg/kg i.p.). The graft was flushed with cold preservation solution (University of Wisconsin [UW]) and either directly transplanted or stored in an ice bath in preservation solution for a defined period of time, and then transplanted. Renal transplantation was performed with donor renal artery, vein, and ureter anastomosed to the recipient's common iliac artery, common iliac vein, and bladder, with a standardized time to reperfusion of 30 minutes, during which the kidney was kept cool with iced gauze. The native kidneys were left in situ for 5 days and removed under inhalational isoflurane anesthetic. Warm IRI experiments in the transplanted kidney were performed as outlined above, with microvascular clamping of the transplanted renal pedicle (26).

BUN measurement. Plasma concentrations of BUN were assessed using an i-STAT Portable Clinical Analyzer with 6+ cartridges (Abbott Laboratories). These cartridges have a maximum BUN reading of $140 \mathrm{mg} / \mathrm{dl}$.

Tissue collection and histopathology. Under terminal general anesthesia, the left kidney in warm IRI experiments or the transplanted kidney in cold IRI experiments was harvested typically 28 days after the ischemia experiment or in select experiments within a shorter time frame as stipulated in the Results, fixed in 10\% neutral-buffered formalin, and paraffin embedded. Histologic sections $(4 \mu \mathrm{m})$ were stained with $\mathrm{H} \& \mathrm{E}$ and trichrome and reviewed by a pathologist (TRB) blinded to the treatment conditions. In the trichrome sections, acute tubular injury, including acute epithelial damage, protein casts, luminal inflammatory cells, tubular atrophy and regeneration, interstitial changes (including interstitial cellularity and inflammation), and interstitial fibrosis were graded on a scale of 0 to 3 . Additional sections were stained as a single group with Sirius red for quantification of fibrosis. Sirius red-stained sections were scanned using the Aperio ScanScope CS slide scanner (Aperio Technologies, Leica Biosystems), and digitized images were analyzed with Aperio ImageScope software using an algorithm optimized for the detection of fibrosis (red staining of collagen fibers) as a percentage of the total volume of tissue on the slide. Subcapsular areas were excluded to limit the analysis to parenchymal changes.

RNA isolation and $q P C R$. RNA extraction and purification were performed using an RNeasy Mini Kit (QIAGEN). RNA was reverse 


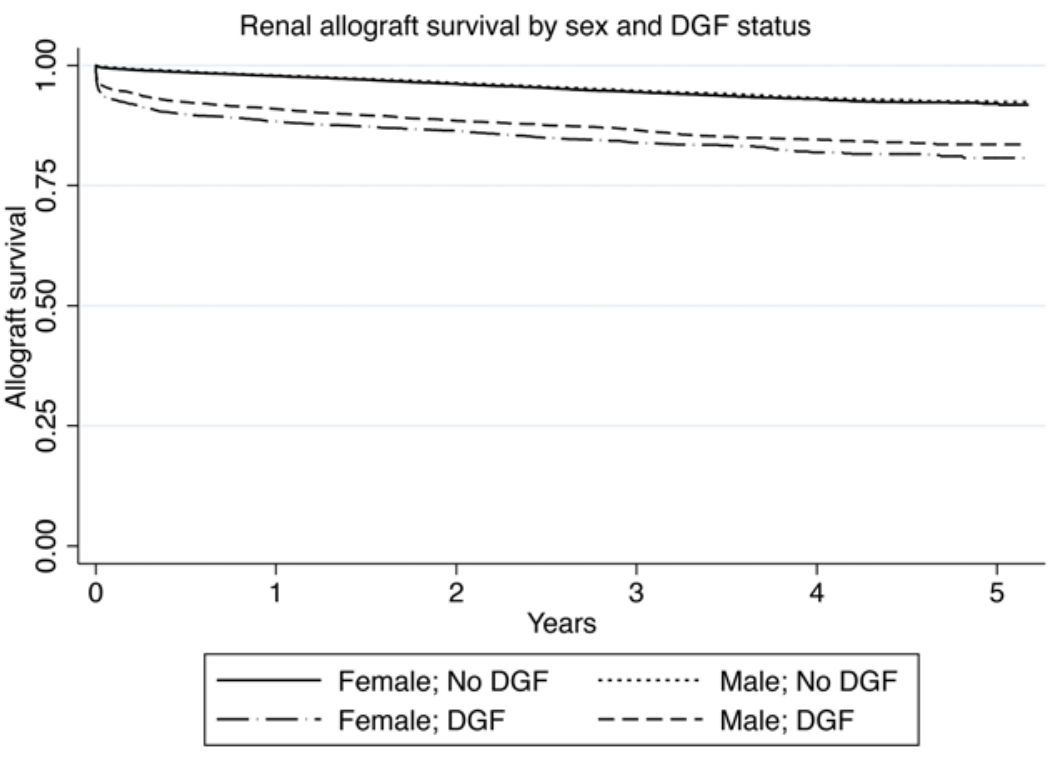

Figure 5. Graft survival by sex and DGF occurrence. Male and female renal transplant recipients who did not develop DGF had equivalent graft survival $(P=0.20)$. Both male and female renal graft recipients with DGF showed decreased graft survival compared with that of recipients of the same sex without DGF $(P<0.01)$. Female renal transplant recipients with DGF had decreased graft survival compared with that of male recipients with DCF $(P=0.002)$.
In human studies, DGF models were constructed using logistic regression with a robust standard error estimate to account for multiple recipients per donor. Demographic comparisons were done using $\chi^{2}$ tests for categorical data and Student's $t$ tests for continuous variables. Statistical analysis was performed with Stata/SE 13.1 software (StataCorp LP). A $P$ value of less than 0.05 was considered statistically significant.

Study approval. All animal studies were approved by the IACUC of Children's Hospital of Philadelphia and performed at a facility accredited by the American Association for Accreditation of Laboratory Animal Care (AALAC).

\section{Author contributions}

DDA, ZW, DRM, YW, RRR, NS, AT, PPR, WWH, and MHL designed the research studies. DDA, ZW, DRM, YW, LW, and MHL conducted the experiments. DDA, ZW, DRM, TRB, YW, GG, RRR, LW, AT, and MHL acquired the data. DDA, ZW, DRM, YW, RRR, PLA, NS, AT, PPR, WWH, and MHL analyzed the data. NS and WWH provided reagents. DDA, TRB, PPR, WWH, and MHL wrote the manuscript. transcribed to cDNA using TaqMan reverse transcription reagents (Applied Biosystems). qPCR was performed using TaqMan Universal PCR Master Mix and gene-specific primers (Applied Biosystems). Samples were run on an ABI PRISM 7000 Sequence Detection System and compared using the $\Delta \Delta \mathrm{Ct}$ method.

Human data collection. Investigation of human renal transplants was performed using information collected from the UNOS database between January 1, 1997, and December 31, 2011. Our analysis included only adult ( $>18$ years of age) kidney recipients who were on dialysis at the time of transplantation, because of the difficulty in defining DGF in patients who did not require renal replacement therapy. Only donors weighing more than $40 \mathrm{~kg}$ and above 10 years of age were included. Information collected and analyzed included donor and recipient sex; donor and recipient age; donor and recipient race; ECD status; DCD status; recipient diabetes status; donor kidney laterality; recipient PRA percentage; cold ischemic time; donor and recipient BW; donor and recipient BMI; DGF status; and 1-, 3-, and 5-year graft survival rates.

Statistics. In animal studies, BUN curves were compared using 2-way ANOVA with Tukey's post-hoc tests as appropriate. The Sirius red staining percentage by group was compared pairwise using a 2-tailed Student's $t$ test and among multiple groups using 1-way ANOVA with Tukey's post-hoc tests. Survival was determined by Kaplan-Meier analysis. Statistical analysis was performed with GraphPad Prism, version 6.0h (GraphPad Software).

\section{Acknowledgments}

Research reported in this publication was supported by the National Institute of Diabetes and Digestive and Kidney Diseases of the NIH under award number 1K08DK092282-01 and the Penn Department of Surgery and Penn Transplant Institute (to M.H. Levine). This work was supported in part by Health Resources and Services Administration contract 234-2005-37011C. The content is the responsibility of the authors alone and does not necessarily reflect the views or policies of the Department of Health and Human Services, nor does mention of trade names, commercial products, or organizations imply endorsement by the US Government. The data reported here have been supplied by UNOS as the contractor for the Organ Procurement and Transplantation Network (OPTN). The interpretation and reporting of these data are the responsibility of the author(s) and in no way should be seen as an official policy of or interpretation by the OPTN or the US Government. The generous contribution of $\mathrm{ER} \alpha-\mathrm{KO}$ mice on a $\mathrm{C} 57 \mathrm{~B} / 6$ background by the laboratory of José Conejo-Garcia at the Wistar Institute is greatly appreciated. The contribution of Shayan Cheraghlou to the development of protocols related to this work is acknowledged.

Address correspondence to: Matthew H. Levine, 3400 Spruce Street, $1^{\text {st }}$ Floor Founder's Building, Transplant Surgery, University of Pennsylvania, Philadelphia, Pennsylvania 19104, USA. Phone: 215.662.7367; E-mail: matthew.levine@uphs.upenn.edu.
1. McGee J, et al. Donor-recipient gender and size mismatch affects graft success after kidney transplantation. J Am Coll Surg. 2010;210(5):718-725.

2. Gratwohl A, Dohler B, Stern M, Opelz G. H-Y as a minor histocompatibility antigen in kidney transplantation: a retrospective cohort study. Lancet.
2008;372(9632):49-53

3. Zhou JY, Cheng J, Huang HF, Shen Y, Jiang Y, Chen JH. The effect of donor-recipient gender mismatch on short- and long-term graft survival in kidney transplantation: a systematic review and meta-analysis. Clin Transplant.
2013;27(5):764-771.

4. Kim SJ, Gill JS. H-Y incompatibility predicts short-term outcomes for kidney transplant recipients. J Am Soc Nephrol. 2009;20(9):2025-2033.

5. Siedlecki A, Irish W, Brennan DC. Delayed graft function in the kidney transplant. Am J Trans- 
plant. 2011;11(11):2279-2296.

6. Ojo AO, Port FK, Wolfe RA, Mauger EA, Williams L, Berling DP. Comparative mortality risks of chronic dialysis and cadaveric transplantation in black end-stage renal disease patients. Am J Kidney Dis. 1994;24(1):59-64.

7. Alves MG, Oliveira PJ, Carvalho RA. Substrate selection in hearts subjected to ischemia/reperfusion: role of cardioplegic solutions and gender. NMR Biomed. 2011;24(9):1029-1037.

8. Ardelt AA, et al. Estradiol modulates post-ischemic cerebral vascular remodeling and improves long-term functional outcome in a rat model of stroke. Brain Res. 2012;1461:76-86.

9. Eckhoff DE, Bilbao G, Frenette L, Thompson JA, Contreras JL. 17- $\beta$-estradiol protects the liver against warm ischemia/reperfusion injury and is associated with increased serum nitric oxide and decreased tumor necrosis factor-alpha. Surgery. 2002;132(2):302-309.

10. Gabel SA, Walker VR, London RE, Steenbergen C, Korach KS, Murphy E. Estrogen receptor beta mediates gender differences in ischemia/reperfusion injury. J Mol Cell Cardiol. 2005;38(2):289-297.

11. Peng X, et al. Gender differences affect blood flow recovery in a mouse model of hindlimb ischemia. Am J Physiol Heart Circ Physiol. 2011;300(6):H2O27-H2O34.

12. Kher A, Meldrum KK, Wang M, Tsai BM, Pitcher JM, Meldrum DR. Cellular and molecular mechanisms of sex differences in renal ischemia-reperfusion injury. Cardiovasc Res. 2005;67(4):594-603.

13. Kher A, et al. Sex differences in the myocardial inflammatory response to acute injury. Shock. 2005;23(1):1-10.

14. Hu H, Wang G, Batteux F, Nicco C. Gender differences in the susceptibility to renal ischemia-reperfusion injury in BALB/c mice. Tohoku J Exp Med. 2009;218(4):325-329.

15. Muller V, et al. Sex hormones and gender-related differences: their influence on chronic renal allograft rejection. Kidney Int . 1999;55(5):2011-2020.

16. Muller V, et al. Sexual dimorphism in renal ischemia-reperfusion injury in rats: possible role of endothelin. Kidney Int . 2002;62(4):1364-1371.

17. Park KM, Kim JI, Ahn Y, Bonventre AJ, Bonventre JV. Testosterone is responsible for enhanced susceptibility of males to ischemic renal injury. J Biol Chem. 2004;279(50):52282-52292.

18. Park KM, Cho HJ, Bonventre JV. Orchiectomy reduces susceptibility to renal ischemic injury: a role for heat shock proteins. Biochem Biophys Res Commun. 2005;328(1):312-317.

19. Robert R, Ghazali DA, Favreau F, Mauco G, Hauet T, Goujon JM. Gender difference and sex hormone production in rodent renal ischemia reperfusion injury and repair. J Inflamm (Lond). 2011;8:14.

20. Rusai K, et al. Gender differences in serum and glucocorticoid regulated kinase-1 (SGK-1) expression during renal ischemia/reperfusion injury. Cell Physiol Biochem. 2011;27(6):727-738.

21. Shen SQ, Zhang Y, Xiong CL. The protective effects of 17beta-estradiol on hepatic ischemia-reperfusion injury in rat model, associated with regulation of heat-shock protein expression. JSurg Res. 2007;140(1):67-76.

22. Soljancic A, et al. Protective role of testosterone in ischemia-reperfusion-induced acute kidney injury. Am J Physiol Regul Integr Comp Physiol. 2013;304(11):R951-R958.

23. Wittnich C, Belanger MP, Askin N, Boscarino C, Wallen WJ. Lower liver transplant success in females: gender differences in metabolic response to global ischemia. Transplant Proc. 2004;36(5):1485-1488.

24. Antus B, et al. Effects of progesterone and selective oestrogen receptor modulators on chronic allograft nephropathy in rats. Nephrol Dial Transplant. 2005;20(2):329-335.

25. Antus B, Yao Y, Song E, Liu S, Lutz J, Heemann U. Opposite effects of testosterone and estrogens on chronic allograft nephropathy. Transpl Int. 2002;15(9-10):494-501.

26. Levine MH, et al. Class-specific histone/protein deacetylase inhibition protects against renal ischemia reperfusion injury and fibrosis formation. Am J Transplant. 2015;15(4):965-973.

27. Hutchens MP, Fujiyoshi T, Komers R, Herson PS, Anderson S. Estrogen protects renal endothelial barrier function from ischemia-reperfusion in vitro and in vivo. Am J Physiol Renal Physiol. 2012;303(3):F377-F385.

28. Hutchens MP, et al. Estrogen is renoprotective via a nonreceptor-dependent mechanism after cardiac arrest in vivo. Anesthesiology. 2010;112(2):395-405.

29. Baylis C. Age-dependent glomerular damage in the rat. Dissociation between glomerular injury and both glomerular hypertension and hypertrophy. Male gender as a primary risk factor. J Clin Invest. 1994;94(5):1823-1829.

30. Delle H, Rocha JR, Cavaglieri RC, Vieira JM Jr, Malheiros DM, Noronha IL. Antifibrotic effect of tamoxifen in a model of progressive renal disease. J Am Soc Nephrol. 2012;23(1):37-48.

31. Bae S, Zhang L. Gender differences in cardioprotection against ischemia/reperfusion injury in adult rat hearts: focus on Akt and protein kinase C signaling. J Pharmacol Exp Ther. 2005;315(3):1125-1135.

32. Ostadal B, Ostadal P. Sex-based differences in cardiac ischaemic injury and protection: therapeutic implications. Br J Pharmacol. 2014;171(3):541-554.

33. Li X, Blizzard KK, Zeng Z, DeVries AC, Hurn PD, McCullough LD. Chronic behavioral testing after focal ischemia in the mouse: functional recovery and the effects of gender. Exp Neurol. 2004;187(1):94-104.

34. Babiker FA, Hoteit LJ, Joseph S, Mustafa AS, Juggi JS. The role of $17-\beta$-estradiol in ischemic preconditioning protection of the heart. Exp Clin Cardiol. 2012;17(3):95-100. 